\title{
Neoadjuvant Therapy Using Checkpoint Inhibitors before Radical Cystectomy for Muscle Invasive Bladder Cancer: A Systematic Review
}

\author{
Hadi SHSM ${ }^{1}$, Usama A. Fahmy ${ }^{2}$ (D) Nabil A. Alhakamy ${ }^{3,4}{ }^{\circledR}$, Mohd G. Khairul-Asri ${ }^{5}$ and Omar Fahmy ${ }^{5, *}$ \\ 1 Department of Urology, Royal Cornwall Hospital, Truro TR1 3LJ, UK; hadi.mohsin@nhs.net \\ 2 Department of Pharmaceutics \& Industrial Pharmacy, Faculty of Pharmacy, King Abdulaziz University, \\ Jeddah 21589, Saudi Arabia; uahmedkauedu.sa@kau.edu.sa \\ 3 Advanced Drug Delivery Research Group, Faculty of Pharmacy, King Abdulaziz University, \\ Jeddah 21589, Saudi Arabia; nalhakamy@kau.edu.sa \\ 4 Center of Excellence for Drug Research and Pharmaceutical Industries, King Abdulaziz University, \\ Jedah 21589, Saudi Arabia \\ 5 Department of Urology, Universiti Putra Malaysia (UPM), Serdang 43400, Selangor, Malaysia; \\ khairulasri@upm.edu.my \\ * Correspondence: omarfahmy.ahmed@upm.edu.my; Tel.: +60-38947-2667
}

check for

updates

Citation: SHSM, H.; Fahmy, U.A.; Alhakamy, N.A.; Khairul-Asri, M.G.; Fahmy, O. Neoadjuvant Therapy Using Checkpoint Inhibitors before Radical Cystectomy for Muscle Invasive Bladder Cancer: A Systematic Review. J. Pers. Med. 2021, 11, 1195. https://doi.org/10.3390/ jpm11111195

Academic Editor: Wojciech Krajewski

Received: 29 September 2021

Accepted: 10 November 2021

Published: 13 November 2021

Publisher's Note: MDPI stays neutral with regard to jurisdictional claims in published maps and institutional affiliations.

Copyright: (c) 2021 by the authors. Licensee MDPI, Basel, Switzerland. This article is an open access article distributed under the terms and conditions of the Creative Commons Attribution (CC BY) license (https:/ / creativecommons.org/licenses/by/ $4.0 /)$.

\begin{abstract}
Background: Neoadjuvant chemotherapy is the standard of care before radical cystectomy for muscle invasive bladder cancer. Recently, checkpoint inhibitors have been investigated as a neoadjuvant treatment after the reported efficacy of checkpoint inhibitors in metastatic urothelial carcinoma. Objectives: The aim of this systematic review is to investigate the role of checkpoint inhibitors as a neoadjuvant treatment for muscle invasive bladder cancer before radical cystectomy. Methods: Based on the PRISMA statement, a systematic review of the literature was conducted through online databases and the American Society of Clinical Oncology (ASCO) Meeting Library. Suitable publications were subjected to full-text assessment. The primary outcome of this review was to identify the impact of neoadjuvant immunotherapy on the oncological outcomes and survival benefits. Results: From the retrieved 254 results, 8 studies including 404 patients were included. Complete response varied between $30 \%$ and 50\%. Downstaging varied between $50 \%$ and $74 \%$. $\geq$ Grade 3 AEs were recorded in $8.6 \%$ of patients who received monotherapy with either Atezolizumab or Pembrolizumab. In patients who received combination treatment, the incidence of $\geq$ Grade 3 AEs was $16.3 \%$ for chemoimmunotherapy and $36.5 \%$ for combined immunotherapy. A total of 373 patients (92\%) underwent radical cystectomy. $\geq$ Grade 3 Clavien-Dindo surgical complications were reported in $21.7 \%$ of the patients. One-year overall survival (OS) and relapse-free survival (RFS) varied between $81 \%$ and $92 \%$, and $70 \%$ and $88 \%$, respectively. Conclusion: The evidence on the use of immune checkpoint inhibitors in the setting of pre-radical cystectomy is quite limited, with noted variability within published trials. Combination with chemotherapy or another checkpoint inhibitor may boost response, although prospective studies with extended follow-up are needed to report on the survival advantages.
\end{abstract}

Keywords: bladder cancer; immunotherapy; checkpoint inhibitors; radical cystectomy; chemotherapy

\section{Introduction}

Muscle invasive bladder cancer remains one of the most lethal forms of urothelial carcinoma. Untreated, the 5-year overall survival rate is less than 5\% [1]. Despite the advancements in peri-operative treatment regimens including neoadjuvant and adjuvant treatment, coupled with timely radical cystectomy (RC) and lymph node dissection, a significant proportion of patients eventually develop metastatic disease [2].

The use of immune mediated treatment in non-muscle invasive bladder cancer has been well documented with the widespread use of Bacillus Calmette-Guérin (BCG) instil- 
lation, BCG treatment, first introduced by Morales in 1976 [3]. Via intravesical instillation of BCG, infiltration of cytotoxic T lymphocytes (CTLs) and cell-mediated cytotoxicity against bladder tumors occurs through activation of innate and adaptive immunity [4]. This formed the basis for immunological manipulation of the bladder mucosa in preventing the recurrence of high-risk non-muscle invasive urothelial carcinoma.

The use of platinum-based chemotherapy as a neoadjuvant treatment prior to cystectomy is well established and common practice [5]. The combination of gemcitabine and cisplatin is the standard first line therapy in the metastatic setting with improvement in median survival of up to 14 months compared to just 6 months [6]. Indeed, numerous trials have elucidated the significant overall survival benefit of the use of neoadjuvant platinum-based chemotherapy in invasive urothelial carcinoma [7].

Recent developments with immune checkpoint inhibitors (ICIs) have shown efficacy in locally advanced and metastatic urothelial carcinoma [8]. A few years ago, the Food and Drug Administration (FDA) approved five immune checkpoint inhibitors (ICIs) for use in various contexts, including first line and second-line therapy for metastatic urothelial carcinoma. Pembrolizumab, a programmed death -1 (PD-1) inhibitor, was recently licensed for high-risk Bacillus Calmette-Guérin (BCG)-unresponsive non-muscle invasive bladder cancer (NMIBC) employing immunotherapy at an early stage of the illness [9]. Very recently, Nivolumab was approved as an adjuvant treatment for high-risk muscle invasive urothelial carcinoma, after showing a better disease-free survival rate compared to the placebo [10].

Whereas numerous trials in the perioperative setting are currently continuing, the role of immune checkpoint inhibition in the neoadjuvant setting is still not clear. This review aims to systematically appraise the emerging role of immune checkpoint inhibition in the neoadjuvant setting for muscle invasive urothelial carcinoma in terms of efficacy and tolerability.

\section{Materials and Methods}

\subsection{Search Strategy}

Based on the PRISMA statement [11], a systematic online search was conducted through online databases (PubMed, EMBASE, Web of Science, Wiley online library and Cochrane databases), in addition to the American Society of Clinical Oncology (ASCO) Meeting Library. The following keywords were used: bladder cancer, radical cystectomy, neoadjuvant, chemotherapy, immunotherapy, checkpoint inhibitors, anti-CTLA-4, and anti-PD-L. An initial assessment of titles and abstracts of all retrieved results was performed with subsequent exclusion of the unrelated articles, case reports, editorials, and review articles. Eligible publications were subjected to full-text assessment followed by exclusion of duplications and other articles unrelated directly to the topic. In addition, a manual search was performed in the references list of the selected papers to avoid missing any eligible publication.

\subsection{Data Extraction}

The following variables were extracted: total number of patients, number of the patients who underwent RC, gender, age, follow-up duration, type of neoadjuvant treatment, adverse events (AEs), RC complications, and survival data. For quantitative analysis, the number of events and total number of each subgroup were extracted. Data were extracted independently by two authors, then a double-check was performed for accuracy.

\subsection{Primary Outcomes}

The primary outcome of this analysis was the impact of neoadjuvant immunotherapy on the outcome of radical cystectomy, in terms of complete response (CR), downstaging (DS), tolerability and AEs, survival benefits, and factors associated with higher response. CR was defined as pT0, meanwhile, DS was defined as $\leq$ pT1. 


\subsection{Statistical Analysis}

The Nordic Cochrane Centre, The Cochrane Collaboration, Copenhagen, employed Review Manager (RevMan) software version 5.4 for statistical analysis and the creation of forest plots for the quantitative analysis and calculation of the odds ratio (OR) and $95 \%$ confidence interval (CI). The $I^{2}$ value was used to determine the heterogeneity of the research. For $I^{2}<50 \%$, a fixed effect model was used, but for $I^{2} \geq 50 \%$, a random effect model was examined. The Z-test was used to assess the overall impact. A $p$-value $<0.05$ was employed as the significance level.

\subsection{Risk of Bias Assessment}

The Newcastle-Ottawa Scale (NOS) was employed in this meta-analysis to assess the quality of non-randomized trials [12]. Scores of 7-9, 4-6, and less than 4 were classified as having a low, moderate, or high risk of bias, respectively.

\section{Results}

\subsection{Search Results}

An initial search retrieved 254 results, which underwent assessment to identify the eligible publications. Finally, 8 studies ( 4 papers and 4 abstracts), including 404 patients were included [13-20]. The screening and selection processes are demonstrated in Figure 1. Different protocols were used in those studies; Atezolizumab (MPDL3280 A) was only investigated as a monotherapy [13]. Pembrolizumab was investigated as a monotherapy [14], or in combination with chemotherapy [18]. Three studies investigated Durvalumab in combination with other drugs, with chemotherapy in two studies $[15,16]$ and with Termelimumab (anti-CTLA-4) in the one study [20]. Nivolumab was investigated in two studies, in combination with chemotherapy [17] and in combination with Ipilimumab (anti-CTLA-4) [19]. A summary of the studies and patients' criteria is presented in Table 1. Based on NOS bias assessment, all the included studies had moderate risk of bias. (Table 2).

\subsection{Oncological Response}

Reported CR varied between $30 \%$ and $50 \%$ among the studies $[15,16]$. CR in monotherapy was slightly lower compared to combination therapy. In 209 patients treated with either Atezolizumab or Pembrolizumab, the average CR was 34\% [13,14]. In 103 patients from three studies, combination of immunotherapy and chemotherapy displayed CR in $40 \%[15,16,18]$. Furthermore, combined immunotherapy via combination of PD-1 drugs or PD-L1 with anti-CTLA-4 showed CR in $40 \%$ in 52 patients from two studies [19,20].

DS varied between $50 \%$ and $74 \%[15,16]$. In 114 patients treated with Pembrolizumab, DS was 55\% [14]. In four studies investigating combined immuno-chemotherapy, $60 \%$ of 144 patients showed DS [15-18], and in a combination of PD-1 agents or PD-L1 with anti-CTLA-4, DS was $58 \%$ in 52 patients [19,20]. Quantitative analysis of the possibility of DS including all studies displayed an OR of 1.63 (0.81-3.28) (Figure 2).

\subsection{Safety and Side Effects}

There was a variation in reporting the AEs. Most of the studies reported on serious AEs only ( $\geq$ Grade 3 ) [15-17,19]. Three studies reported on all AEs $[13,14,20]$. $\geq$ Grade 3 AEs varied between $3 \%$ and $55 \%[15,19]$. In patients who received monotherapy with either Atezolizumab or Pembrolizumab, $\geq$ Grade 3 AEs were recorded in $8.6 \%$ [13,14]. Meanwhile, combination therapy was reported to have higher $\geq$ Grade 3 AEs. In 104 patients who received combined immune and chemotherapy, the incidence of $\geq$ Grade 3 AEs was $16.3 \%$ [15-17], and in 52 patients who received combined PD-1 or PD-L1 with antiCTLA-4, it was $36.5 \%[19,20]$. Figure 3 displays the comparison between mono and combined therapy in outcome and $\geq$ Grade 3 AEs. 
Online search through online data bases (PubMed, EMBASE, Web of Science, Wiley online library and Cochrane databases) \& (ASCO) Meeting Library

$$
(n=254)
$$

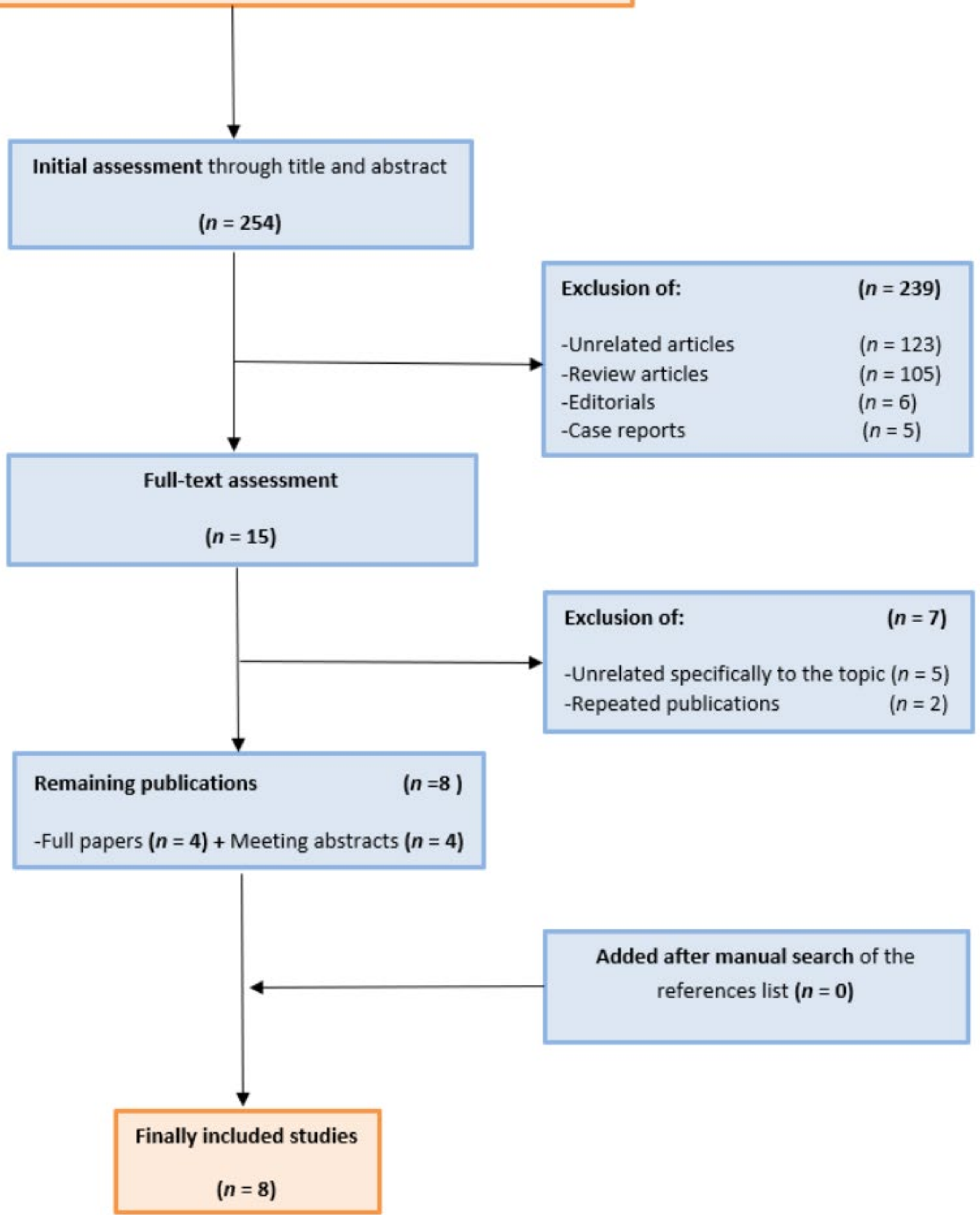

Figure 1. CONSORT diagram for the screening and selection processes of the included studies. 
Table 1. Summary of the included studies.

\begin{tabular}{|c|c|c|c|c|c|c|c|}
\hline Study & Country & Trial Name & Type & Neoadjuvant Treatment & No. of Patients & Male/Female & Mean Age \\
\hline Powles 2019 [13] & UK & ABACUS & single-arm phase II & Atezolizumab & 95 & $81 / 14$ & 73 \\
\hline Necchi 2020 [14] & Italy & PURE-01 & single-arm phase II & Pembrolizumab & 114 & $99 / 15$ & 66 \\
\hline Cathomas 2020 [16] & Switzerland & SAKK $-06 / 17$ & single-arm phase II & Durvalumab + Chemo & 34 & $27 / 7$ & 70 \\
\hline Gupta 2020 [17] & USA & BLASST-1 & single-arm phase II & Nivolumab + Chemo & 41 & - & - \\
\hline Hoimes 2020 [18] & USA & - & 3-arm Phase Ib/II & Pembrolizumab + Chemo & 40 & $30 / 10$ & 65 \\
\hline Dijk 2020 [19] & Netherland & NABUCCO & single-arm phase I & Nivolumab + Ipilimumab & 24 & $18 / 6$ & $65(50-81)$ \\
\hline Gao 2020 [20] & USA & - & single-arm phase I & Durvalumab + Termelimumab & $27^{*}$ & $20 / 8$ & $71(24-83)$ \\
\hline
\end{tabular}

Table 2. Newcastle-Ottawa Scale for risk of bias assessment of the included studies (scores $\geq 7-9,4-6,<4$ are considered as low, intermediate, and high risk, respectively).

\begin{tabular}{|c|c|c|c|c|c|c|c|c|}
\hline \multirow[b]{2}{*}{ Study } & \multicolumn{4}{|c|}{ Selection } & \multirow{2}{*}{$\begin{array}{l}\text { Comparability } \\
\\
\begin{array}{c}\text { Assessment of } \\
\text { Outcome }\end{array}\end{array}$} & \multicolumn{2}{|l|}{ Outcome } & \multirow[t]{2}{*}{ Overall } \\
\hline & $\begin{array}{l}\text { Representativeness } \\
\text { of Exposed Cohort }\end{array}$ & $\begin{array}{l}\text { Selection of } \\
\text { Nonexposed }\end{array}$ & $\begin{array}{l}\text { Ascertainment } \\
\text { of Exposure }\end{array}$ & $\begin{array}{l}\text { Outcome not } \\
\text { Present at Start }\end{array}$ & & $\begin{array}{c}\text { Adequate } \\
\text { Follow-up Length }\end{array}$ & $\begin{array}{l}\text { Adequacy of } \\
\text { Follow-up }\end{array}$ & \\
\hline Powles 2019 [13] & * & & * & * & * & * & * & $6 / 9$ \\
\hline Necchi 2020 [14] & * & & * & * & * & * & * & $6 / 9$ \\
\hline Moreno 2020 [15] & * & & * & * & * & * & & $5 / 9$ \\
\hline Cathomas 2020 [16] & * & & * & * & * & * & & $5 / 9$ \\
\hline Hoimes 2020 [18] & * & & * & * & * & * & & $5 / 9$ \\
\hline Dijk 2020 [19] & * & & * & * & * & * & * & $6 / 9$ \\
\hline Gao 2020 [20] & * & & * & * & * & * & * & $6 / 9$ \\
\hline
\end{tabular}

* One star for each item except the comparability is assessed by 2 stars (total score is 9 stars). 


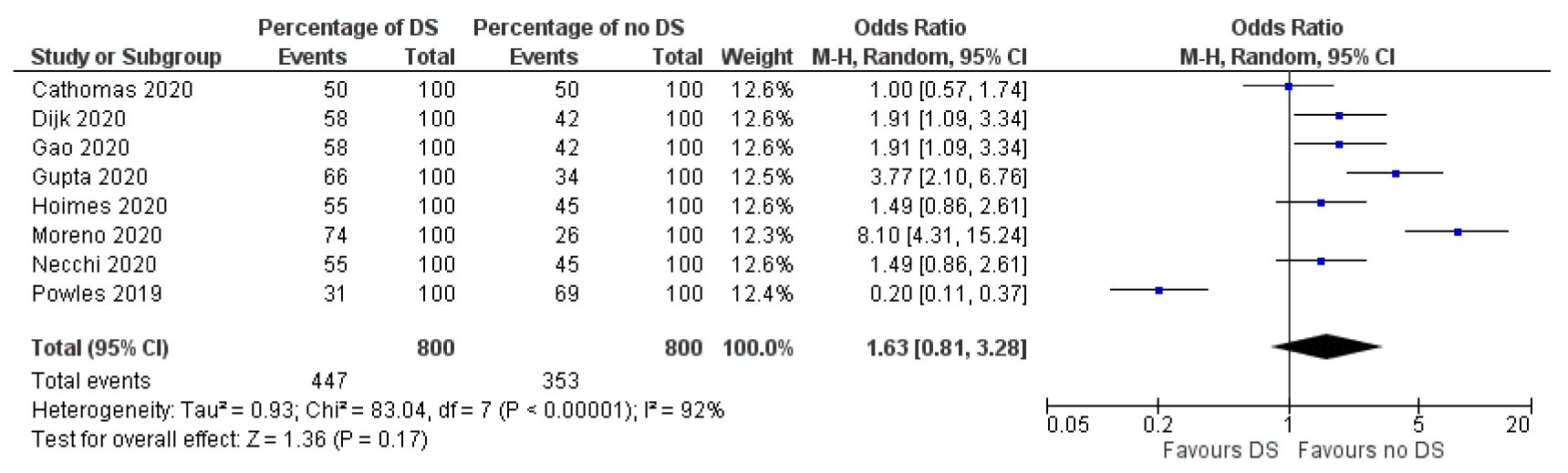

Figure 2. Forest plot for the quantitative analysis of odds ratio for possibility of downstaging (DS).

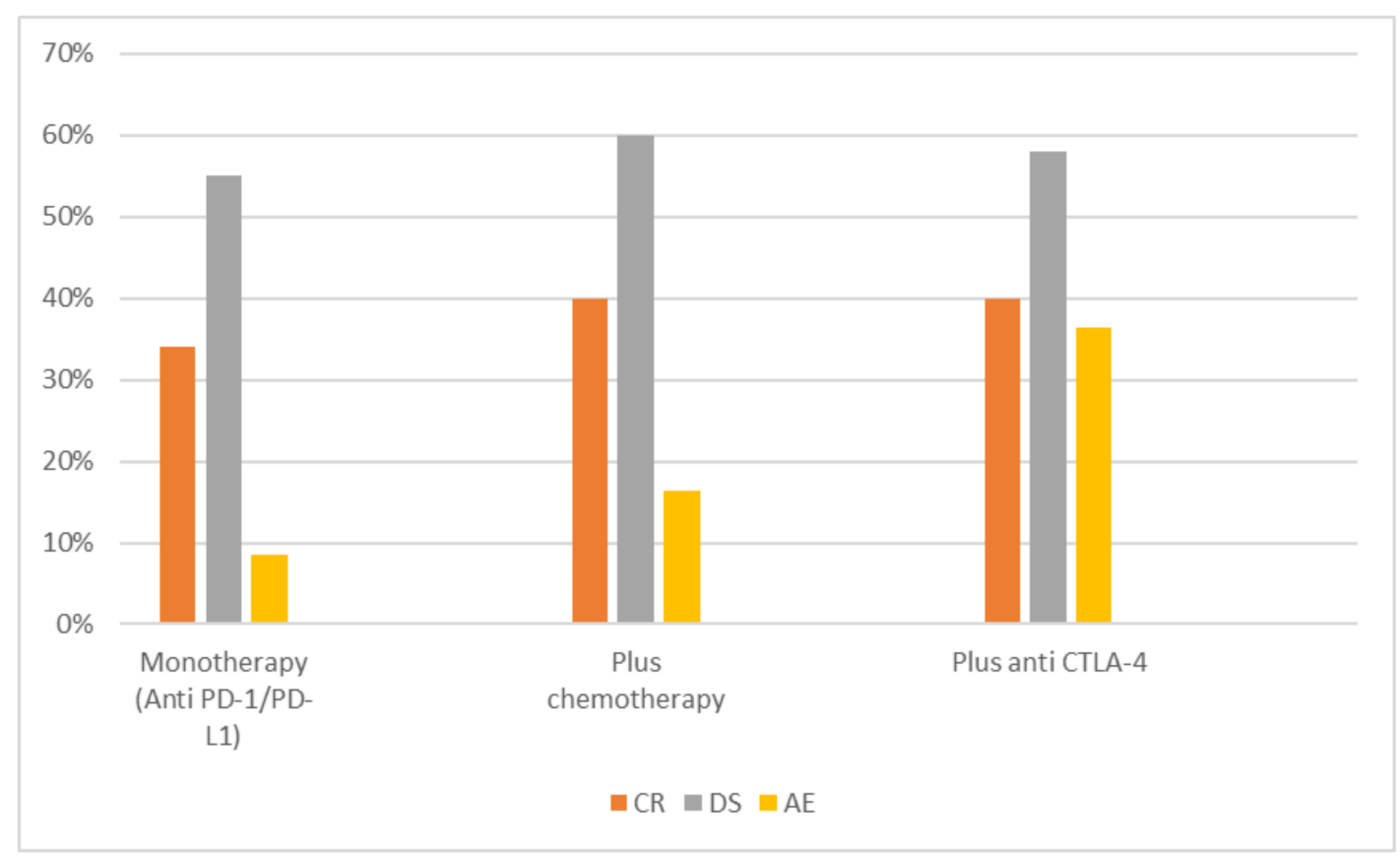

Figure 3. Comparison of the incidence of complete response (CR), downstaging (DS) and adverse events (AE) between anti-PD-1/PD-L1 as monotherapy or in combination with chemotherapy or anti-CTLA-4.

\subsection{Surgical Complications}

Of the 404 patients included in this review, 373 (92\%) underwent radical cystectomy. Surgical complications were reported in five studies [13-16,20]. Overall incidence of surgical complications was 56\% (142/253 patients) $[13,14,16,20]$. $\geq$ Grade 3 Clavien-Dindo surgical complications were reported in $21.7 \%$ (54/249 patients) [13-16,21].

\subsection{Survival Outcomes}

There are no available data on long-term survival outcomes. Only 1-year overall survival (OS) and relapse free survival (RFS) were reported in 4 studies, and varied between $81 \%$ and $92 \%$, and $70 \%$ and $88 \%$, respectively $[13,18-20]$. A summary of the outcome of the included studies is provided in Table 3. 
Table 3. Summary of the outcome of the included studies.

\begin{tabular}{|c|c|c|c|c|c|c|c|c|c|}
\hline \multirow{2}{*}{ Study } & \multirow{2}{*}{ DS } & \multirow{2}{*}{ CR } & \multicolumn{2}{|c|}{$\mathrm{AE}(\%)$} & \multirow{2}{*}{ RC (\%) } & \multicolumn{2}{|c|}{ Surgical Complications (\%) } & \multirow{2}{*}{1 y OS } & \multirow{2}{*}{1 y RFS } \\
\hline & & & All Grades & $\geq$ Grade 3 & & All Grades & $\geq$ Grade 3 & & \\
\hline $\begin{array}{l}\text { Powles } \\
2019 \text { [13] }\end{array}$ & - & $31 \%$ & $49 / 95(52)$ & $10 / 95(11)$ & $87 / 95(92)$ & $54 / 87(62)$ & $15 / 87(17)$ & - & $79 \%$ \\
\hline $\begin{array}{c}\text { Necchi } \\
2020[14]\end{array}$ & $55 \%$ & $37 \%$ & $85 / 114(75)$ & 8/114 (7) & $112 / 114(98)$ & $69 / 112(62)$ & $26 / 112(23)$ & - & - \\
\hline $\begin{array}{l}\text { Moreno } \\
2020 \text { [15] }\end{array}$ & $74 \%$ & $50 \%$ & NA & $1 / 29(3)$ & $26 / 29(90)$ & NA & $5 / 20(25)$ & - & - \\
\hline $\begin{array}{c}\text { Cathomas } \\
2020 \text { [16] }\end{array}$ & $50 \%$ & $30 \%$ & NA & $8 / 34(24)$ & $30 / 34(88)$ & $13 / 30(43)$ & $8 / 30(27)$ & - & - \\
\hline $\begin{array}{c}\text { Gupta } \\
2020 \text { [17] }\end{array}$ & $66 \%$ & - & NA & $8 / 41(20)$ & $40 / 41(98)$ & NA & NA & - & - \\
\hline $\begin{array}{l}\text { Hoimes } \\
2020[18]\end{array}$ & $55 \%$ & $44 \%$ & NA & NA & $36 / 40(90)$ & NA & NA & $94 \%$ & $80 \%$ \\
\hline $\begin{array}{c}\text { Dijk } \\
2020 \text { [19] }\end{array}$ & $58 \%$ & $46 \%$ & NA & 13/24 (55) & $24 / 24(100)$ & NA & NA & $92 \%$ & $88 \%$ \\
\hline $\begin{array}{c}\text { Gao } \\
2020[20]\end{array}$ & $58 \%$ & $37 \%$ & 25/27 (93) & 6/27 (21) & $23 / 27(86)$ & $5 / 23(22)$ & NA & $88 \%$ & $82 \%$ \\
\hline
\end{tabular}

DS: downstaging; CR: complete response; AE: adverse events; RC: radical cystectomy; OS: overall survival; RFS: recurrence-free survival.

\subsection{Subgroup Quantitative Analysis}

Subgroup quantitative analysis was feasible to assess the CR based on the clinical stage and PD-L1 positivity. In comparing patients with cT2 disease and patients with cT3 or cT4, CR was achieved in $32 \%(26 / 81)$ and $21.6 \%$ (8/37), respectively. However, the difference was not significant $(\mathrm{OR}=1.82 ; \mathrm{Z}=1.24 ; p=0.22)$ (Figure $4 \mathrm{a})$. When cT4 patients were compared to cT2 or cT3, the CR rate was $20 \%(2 / 10)$ for cT4 vs $28.7 \%(31 / 108)$ $(\mathrm{OR}=1.59 ; \mathrm{Z}=0.58 ; p=0.57)[13,20]$ (Figure $4 \mathrm{~b})$. The risk ratio $(\mathrm{RR})$ of developing $\geq$ Grade 3 AEs due to combination of Durvalumab and chemotherapy was calculated from the two studies using the same combination and showed a RR of $0.10(0.01-1.65)[15,16]$ (Figure 5).

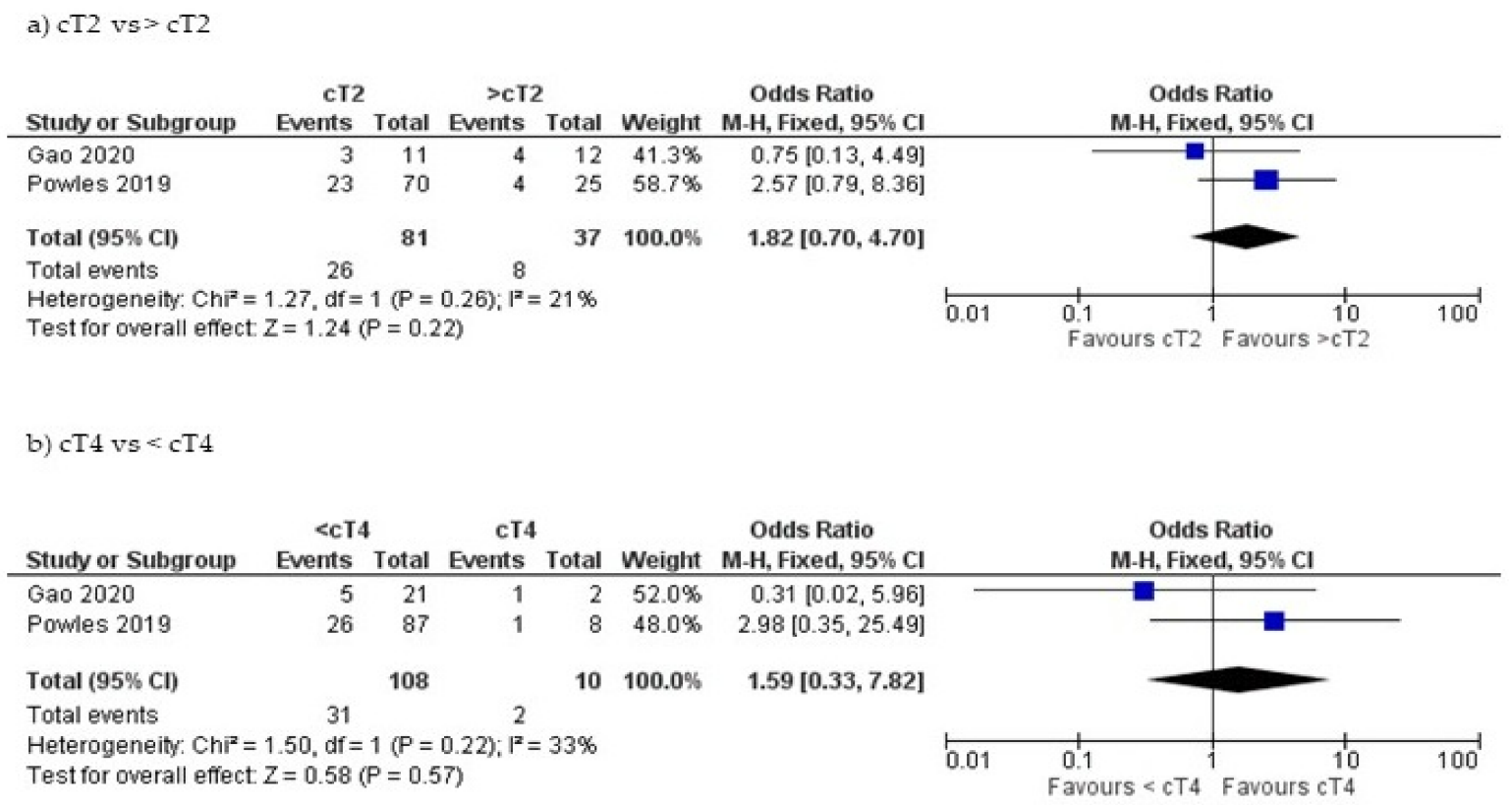

Figure 4. Forest plot for the impact of clinical stage of bladder cancer on complete response rate. (a) cT2 vs. > cT2, (b) cT4 vs. < cT4. 


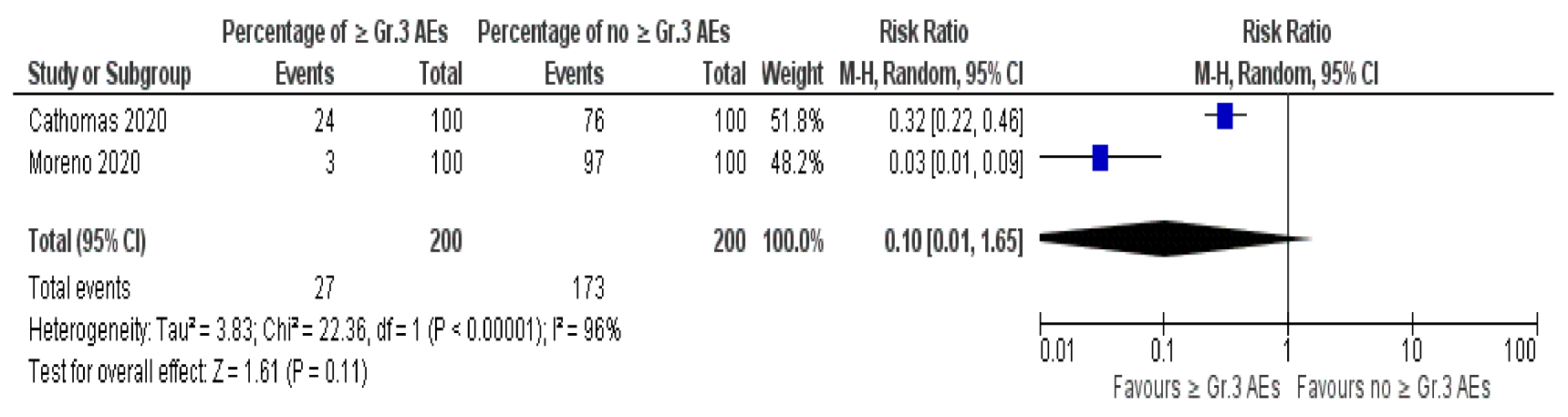

Figure 5. Forest plot of the risk ratio of developing $\geq$ Grade 3 adverse events due to combination of Durvalumab and chemotherapy.

Investigation of CR based on PD-L1 positivity displayed a trend towards higher CR in PD-L1 positive patients, yet it did not reach the significance level. CR was $43.2 \%(32 / 74)$ in positive patients vs $22.5 \%(16 / 71)$ in negative patients. $(\mathrm{OR}=2.94 ; \mathrm{Z}=1.33 ; p=0.18)$ (Figure 6) [13,22].

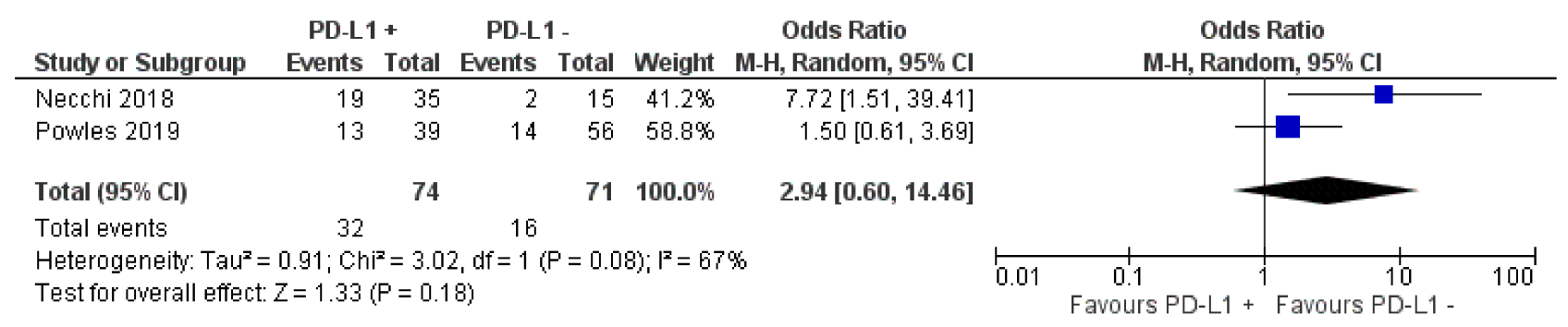

Figure 6. Forest plot for the impact of PD-L1 positivity on complete response rate.

NB: Necchi 2018 [22] is the early report for the PURE-01 trial as the data included in this forest plot was not available in Necchi 2020 [14].

\section{Discussion}

It has been reported that in the setting of urothelial carcinoma, advanced and aggressive tumors with poor survival outcomes are seen in cancers which express high levels of programmed death-ligand 1 (PD-L1) expression [23]. It is postulated that the antitumor immune response is improved by ICIs due to a greater T cell-mediated antitumor immune response which is elicited by the greater availability of neoantigens [24].

Atezolizumab became the first new drug approved in metastatic urothelial carcinoma in over 30 years followed by nivolumab in 2017 [25]. This has sparked interest in the possible role of immune checkpoint inhibitors in the neoadjuvant setting. Current trials find a role for immune checkpoint inhibitors in the neoadjuvant setting for patients who are contraindicated for platinum-based therapy mainly due to renal impairment [25].

Unlike standard neoadjuvant combination chemotherapy, the role of neoadjuvant PDL1 inhibitors in muscle invasive bladder cancer is not well established. Some preliminary studies have looked at the role of PD-L1 inhibitors as a neoadjuvant treatment, in a select group of patients who are unfit or did not respond to chemotherapy [14].

In our systematic review, we looked at the role of PD-L1 inhibitors in the neoadjuvant setting. Two broad categories were identified; the use of PD-L1 inhibitors as monotherapy or in combination with either CTL-4 inhibitors or chemotherapy.

In terms of survival, we observed that combination chemotherapy and PD-L1 inhibitors showed only a modest improvement in complete response compared to PD-L1 inhibitor monotherapy or combination therapy. This may call into question the rationale of combination therapy, with its attendant higher risk of toxicity and also costs in this setting. 
The downstaging was comparable between all three categories of patients: having either PD-L1 inhibitor monotherapy. In combination with chemotherapy or combination with a CTL-4, downstaging was variable across all groups.

Regardless of this variable response, the results are promising, especially for the subset of patients who may not be suitable for neoadjuvant chemotherapy. The role of neoadjuvant treatment in the setting of muscle invasive bladder cancer is well established and proven to reduce relapse and improve survival by eliminating micro-metastatic deposits prior to radical cystectomy [26]. In all the studies reviewed, an average of $90 \%$ of patients went on to have a radical cystectomy.

An interesting observation was seen in the analysis of safety and adverse events. Inhibition of autoimmunity and limitation of immune activation which occurs with administration of these medications are thought to contribute to a wide range of side effects resembling autoimmune reactions. The rationale for dual immune checkpoint inhibition lies in its potential for synergistic immunotherapy activity and thus efficacy [27].

Whilst it was not surprising to see a higher reported rate of side effects and surgical complications in the combination of chemotherapy and PD-L1 versus PD-L1 monotherapy, the studies looking at combination of PD-L1 and CTL- 4 consistently reported a higher complication rate.

In terms of survival, only four studies looked into the analysis of recurrence-free survival and overall survival. As the role of immune checkpoint inhibition in the neoadjuvant setting of muscle invasive bladder cancer is still in its infancy, this is not surprising as insufficient time has lapsed to allow results to accrue. Nevertheless, RFS in the region above $70 \%$ and OS of over $80 \%$ across all studies look to be very promising. Furthermore, based on a recent systematic review and meta-analysis by Fahmy et al., complete downstaging to T0 before radical cystectomy is associated with better survival outcomes [2]. Therefore, the observed CR and DS in this review could be reflected in better survival in the future.

This study is limited by the wide disparity between the included studies in the various treatment regiments and combinations of therapeutic agents. These might carry additional confounders for the observations seen. Furthermore, all the studies are phase I or II, with a small number of patients and lacking a control arm for comparison. All that limited the chance for meaningful quantitative analysis for pooled outcomes. In addition, all patients are from the USA or Europe, which might not be reflective of the global ethnical variations. However, from a practical point of view, adequate global participation can be limited by the availability of drugs and the difference between national health insurance systems among the countries. It will also be particularly interesting to see long-term survival data to allow more direct comparison between the use of immune checkpoint inhibition versus standard chemotherapy in this setting.

We have no doubt that as the role of immune checkpoint inhibition expands across all types of solid cancers and there is longer data accrual, its role in the neoadjuvant setting may become more established. Nevertheless, this review suggests the promising role it currently has in the expansion of our armamentarium in fighting muscle invasive bladder cancer.

\section{Conclusions}

The data on application of immune checkpoint inhibitors in the pre-radical cystectomy setting is very limited with heterogeneity observed among published studies. Combination with chemotherapy or other checkpoint inhibitors might improve the response; however, prospective trials with longer follow-up is required to report on the survival benefits. Identification of selection criteria for patients who can maximally benefit from this treatment modality ought to be aimed for in future trials.

Author Contributions: Conceptualization, O.F.; N.A.A.; H.S.; writing-original draft preparation, O.F.; H.S.; writing—review and editing, O.F.; H.S.; U.A.F.; visualization, O.F.; M.G.K.-A.; supervision, O.F.; project administration, N.A.A.; funding acquisition, U.A.F.; N.A.A. All authors have read and agreed to the published version of the manuscript. 
Funding: The authors gratefully acknowledge The Deanship of Scientific Research (DSR) at King Abdulaziz University, Jeddah, Saudi Arabia for funding this project, under grant number (FP-021-43).

Data Availability Statement: Not applicable.

Conflicts of Interest: The authors declare no conflict of interest. The funders had no role in the design of the study; in the collection, analyses, or interpretation of data; in the writing of the manuscript, or in the decision to publish the results.

\section{References}

1. Martini, A.; Sfakianos, J.P.; Renström-Koskela, L.; Mortezavi, A.; Falagario, U.G.; Egevad, L.; Hosseini, A.; Mehrazin, R.; Galsky, M.D.; Steineck, G.; et al. The natural history of untreated muscle-invasive bladder cancer. BJU Int. 2020, 125, 270-275. [CrossRef] [PubMed]

2. Fahmy, O.; Khairul-Asri, M.G.; Schubert, T.; Renninger, M.; Malek, R.; Kübler, H.; Stenzl, A.; Gakis, G. A systematic review and meta-analysis on the oncological long-term outcomes after trimodality therapy and radical cystectomy with or without neoadjuvant chemotherapy for muscle-invasive bladder cancer. Urol. Oncol. 2018, 36, 43-53. [CrossRef] [PubMed]

3. Morales, A.; Eidinger, D.; Bruce, A.W. Intracavitary Bacillus Calmette-Guerin in the treatment of superficial bladder tumors. J. Urol. 1976, 116, 180-183. [CrossRef]

4. Fahmy, O.; Khairul-Asri, M.G.; Stenzl, A.; Gakis, G. Systemic anti-CTLA-4 and intravesical Bacille-Calmette-Guerin therapy in non-muscle invasive bladder cancer: Is there a rationale of synergism? Med. Hypotheses 2016, 92, 57-58. [CrossRef]

5. Vale, C.L. Neoadjuvant chemotherapy in invasive bladder cancer: Update of a systematic review and meta-analysis of individual patient data advanced bladder cancer (ABC) meta-analysis collaboration. Eur. Urol. 2005, 48, 202. [CrossRef]

6. von der Maase, H.; Sengelov, L.; Roberts, J.T.; Ricci, S.; Dogliotti, L.; Oliver, T.; Moore, M.J.; Zimmermann, A.; Arning, M. Longterm survival results of a randomized trial comparing gemcitabine plus cisplatin, with methotrexate, vinblastine, doxorubicin, plus cisplatin in patients with bladder cancer. J. Clin. Oncol. 2005, 23, 4602-4608. [CrossRef]

7. Yin, M.; Joshi, M.; Meijer, R.P.; Glantz, M.; Holder, S.; Harvey, H.A.; Kaag, M.; Fransen van de Putte, E.E.; Horenblas, S.; Drabick, J.J Neoadjuvant Chemotherapy for Muscle-Invasive Bladder Cancer: A Systematic Review and Two-Step Meta-Analysis. Oncologist 2016, 21, 708-715. [CrossRef]

8. Fahmy, O.; Khairul-Asri, M.G.; Stenzl, A.; Gakis, G. The current status of checkpoint inhibitors in metastatic bladder cancer. Clin. Exp. Metast. 2016, 33, 629-635. [CrossRef]

9. Roviello, G.; Catalano, M.; Santi, R.; Palmieri, V.E.; Vannini, G.; Galli, I.C.; Buttitta, E.; Villari, D.; Rossi, V.; Nesi, G. Immune Checkpoint Inhibitors in Urothelial Bladder Cancer: State of the Art and Future Perspectives. Cancers 2021, 13, 4411. [CrossRef]

10. Bajorin, D.F.; Witjes, J.A.; Gschwend, J.E.; Schenker, M.; Valderrama, B.P.; Tomita, Y.; Bamias, A.; Lebret, T.; Shariat, S.F.; Park, S.H.; et al. Adjuvant Nivolumab versus Placebo in Muscle-Invasive Urothelial Carcinoma. N. Engl. J. Med. 2021, 384, 2102-2114. [CrossRef]

11. Moher, D.; Shamseer, L.; Clarke, M.; Ghersi, D.; Liberati, A.; Petticrew, M.; Shekelle, P.; Stewart, L.A. Preferred reporting items for systematic review and meta-analysis protocols (PRISMA-P) 2015 statement. Syst. Rev. 2015, 4, 1. [CrossRef]

12. Wells, G.A.; Shea, B.; O'Connell, D.; Peterson, J.; Welch, V.; Losos, M.; Tugwell, P. Ottawa Hospital Research Institute. Available online: http:/ / www.ohri.ca/programs/clinical_epidemiology/oxford.asp (accessed on 10 September 2021).

13. Powles, T.; Kockx, M.; Rodriguez-Vida, A.; Duran, I.; Crabb, S.J.; Van Der Heijden, M.S.; Szabados, B.; Pous, A.F.; Gravis, G.; Herranz, U.A. Clinical efficacy and biomarker analysis of neoadjuvant atezolizumab in operable urothelial carcinoma in the ABACUS. Nat. Med. 2019, 25, 1706-1714. [CrossRef]

14. Necchi, A.; Raggi, D.; Gallina, A.; Madison, R.; Colecchia, M.; Lucianò, R.; Montironi, R.; Giannatempo, P.; Farè, E.; Pederzoli, F.; et al. Updated Results of PURE-01 with Preliminary Activity of Neoadjuvant Pembrolizumab in Patients with Muscle-invasive Bladder Carcinoma with Variant Histologies. Eur. Urol. 2020, 77, 439-446. [CrossRef]

15. Rodriguez-Moreno, J.F.; de Velasco, G.; Bravo Fernandez, I. Impact of the Combination of Durvalumab (MEDI4736) Plus Olaparib (AZD2281) Administered Prior to Surgery in the Molecular Profile of Resectable Urothelial Bladder Cancer: NEODURVARIB Trial. J. Clin. Oncol. 2020, 38, 542. [CrossRef]

16. Cathomas, R.; Petrausch, U.; Hayoz, S. Perioperative chemoimmunotherapy with durvalumab (Durva) in combination with cisplatin/gemcitabine (Cis/Gem) for operable muscle-invasive urothelial carcinoma (MIUC): Preplanned interim analysis of a single-arm phase II trial (SAKK 06/17). J. Clin. Oncol. 2020, 20, 499. [CrossRef]

17. Gupta, S.; Sonpavde, G.; Weight, C.J. Results from BLASST-1 (Bladder Cancer Signal Seeking Trial) of nivolumab, gemcitabine, and cisplatin in muscle invasive bladder cancer (MIBC) undergoing cystectomy. J. Clin. Oncol. 2020, 20, 439. [CrossRef]

18. Hoimes, C.J.; Albany, C.; Hoffman-Censits, J. A phase Ib/II study of neoadjuvant pembrolizumab (pembro) and chemotherapy for locally advanced urothelial cancer (UC). Clin. Oncol. 2020, 38, 5047. [CrossRef]

19. Van Dijk, N.; Gil-Jimenez, A.; Silina, K.; Hendricksen, K.; Smit, L.A.; de Feijter, J.M.; van Montfoort, M.L.; van Rooijen, C.; Peters, D.; Broeks, A. Preoperative ipilimumab plus nivolumab in locoregionally advanced urothelial cancer: The NABUCCO trial. Nat. Med. 2020, 26, 1839-1844. [CrossRef] 
20. Gao, J.; Navai, N.; Alhalabi, O.; Siefker-Radtke, A.; Campbell, M.T.; Tidwell, R.S.; Guo, C.C.; Kamat, A.M.; Matin, S.F.; Araujo, J.C. Neoadjuvant PD-L1 plus CTLA-4 blockade in patients with cisplatin-ineligible operable high-risk urothelial carcinoma. Nat. Med. 2020, 26, 1845-1851. [CrossRef]

21. Dindo, D.; Demartines, N.; Clavien, P.A. Classification of surgical complications: A new proposal with evaluation in a cohort of 6336 patients and results of a survey. Ann. Surg. 2004, 240, 205-213. [CrossRef]

22. Necchi, A.; Anichini, A.; Raggi, D.; Briganti, A.; Massa, S.; Lucianò, R.; Colecchia, M.; Giannatempo, P.; Mortarini, R.; Bianchi, M.; et al. Pembrolizumab as Neoadjuvant Therapy Before Radical Cystectomy in Patients with Muscle-Invasive Urothelial Bladder Carcinoma (PURE-01): An Open-Label, Single-Arm, Phase II Study. J. Clin. Oncol. 2018, 36, 3353-3360. [CrossRef] [PubMed]

23. Lopez-Beltran, A.; Cimadamore, A.; Blanca, A.; Massari, F.; Vau, N.; Scarpelli, M.; Cheng, L.; Montironi, R. Immune Checkpoint Inhibitors for the Treatment of Bladder Cancer. Cancers 2021, 13, 131. [CrossRef] [PubMed]

24. Samstein, R.M.; Lee, C.H.; Shoushtari, A.N.; Hellmann, M.D.; Shen, R.; Janjigian, Y.Y.; Barron, D.A.; Zehir, A.; Jordan, E.J.; Omuro, A. Tumor mutational load predicts survival after immunotherapy across multiple cancer types. Nat. Genet. 2019, 51, 202-206. [CrossRef] [PubMed]

25. Davarpanah, N.N.; Yuno, A.; Trepel, J.B.; Apolo, A.B. Immunotherapy: A new treatment paradigm in bladdercancer. Curr. Opin. Oncol. 2017, 29, 184-195. [CrossRef]

26. Raphael, M.J.; Booth, C.M. Neoadjuvant chemotherapy for muscle-invasive bladder cancer: Underused across the 49th parallel. Can. Urol. Assoc. J. 2019, 13, 29-31. [CrossRef]

27. Silva, A.L.; Abreu-Mendes, P.; Martins, D.; Mendes, F. The Impact of Immune Checkpoint-Inhibitors Therapy in Urinary Bladder Cancer. Onco 2021, 1, 3-22. [CrossRef] 